VOL. $18(1978), 46 \mid-464$.

\title{
On the distance of non-reflexive spaces to the collection of all conjugate spaces
}

\section{Ivan Singer}

We show that there exists a conjugate Banach space $E=B^{*}$ with a basis, such that the distance from $E$ to the collection of all conjugate Banach spaces can be made arbitrarily large, by suitable renorming of $E$. This solves a problem raised by B.V. Godun, Dokl. Akad. Nauk SSSR 236 (1977), 18-20.

The Banach-Mazur distance of two Banach spaces $E$ and $F$ is defined by

$$
d(E, F)=\left\{\begin{array}{l}
\inf \|u\|\left\|u^{-1}\right\| \text { if } E \text { is isomorphic to } F, \\
+\infty \text { if } E \text { is not isomorphic to } F,
\end{array}\right.
$$

where the infimum is taken over all isomorphisms $u$ of $E$ onto $F$. Recently, Godun [5] has introduced, for a Banach space $E$, the number (2) $D(E)=\sup _{\|\| \| A} \inf _{X^{*} \in C} d\left((E,\|\cdot\|), X^{*}\right)=\sup _{\|\cdot\| \in A} d((E,\|\cdot\|), \mathcal{C})$,

where $\mathcal{C}$ denotes the collection of all conjugate Banach spaces $X^{*}$ and $A$ denotes the collection of all equivalent norms $\|\cdot\|$ on $E$. Godun has shown ([5], Theorem) that for every non-reflexive Banach space $E$ we have $D(E) \geq 2$ and has raised the following problem: does there exist a conjugate Banach space $E=B^{*}$ such that $D(E)=\infty$ ? In the present note Received 4 April 1978. 
we shall show that the answer is affirmative and, moreover, one can even find such an $E$ with a basis. Our proof is very short, but uses deep results of Enflo [3], Lindenstrauss [8], Figiel and Johnson [4], and Grothendieck [6].

We recall that a Banach space $E$ is said to have

(a) the approximation property, if for every compact subset $Q$ of $E$ and every $\varepsilon>0$ there exists a continuous linear operator $v: E \rightarrow E$ with $\operatorname{dim} v(E)<\infty$, such that $\|x-v(x)\|<\varepsilon \quad(x \in Q)$;

(b) the $\lambda$-approximation property, if one can find $v$ with the above properties and satisfying, in addition, $\|v\| \leq \lambda$.

The 1-approximation property is also called the metric approximation property.

Now we are ready to give the following

EXAMPLE. By Enflo's negative solution of the approximation problem [3] and a result of Lindenstrauss [8], there exists a conjugate Banach space $E=B^{*}$ with a basis, such that $E^{*}=B^{* *}$ does not have the approximation property. Then, by a theorem of Figiel and Johnson [4], there exists a sequence $\left\{\|\| \cdot \|_{n}\right\}_{n=1}^{\infty}$ of equivalent norms on $E$, so that $\left(E,\|\cdot\| \|_{n}\right)$ does not have the $n$-approximation property. Now let $X^{*}$ be any conjugate Banach space.

Case $1^{\circ}$. $X^{*}$ is separable and has the approximation property. Then, as has been observed by Johnson, Rosenthal, and Zippin ([7], Remark 4.31) the results of Grothendieck [6] imply that $X^{*}$ has the metric approximation property. Hence,

$$
d\left(\left(E,\|\cdot\|_{n}\right), X^{*}\right) \geq n \quad(n=1,2, \ldots)
$$

(since otherwise it would follow that $\left(E,\|\cdot\|_{n}\right)$ has the $n$-approximation property).

Case $2^{\circ} . X^{*}$ is separable and does not have the approximation property, or $X^{*}$ is non-separable. In this case,

$$
d\left(\left(E, \quad\|\cdot\| \|_{n}\right), X^{*}\right)=\infty
$$


(since $\left(E,\|\cdot\|_{n}\right)$ has a basis, whence also the approximation property, so $\left(E,\|\| \|_{n}\right)$ is not isomorphic to $\left.X^{*}\right)$.

Hence, since $X^{*}$ was an arbitrary conjugate Banach space, it follows that

$$
\inf _{X^{*} \in C} d\left(\left(E,\|\| \|_{n}\right), X^{*}\right) \geq n \quad(n=1,2, \ldots),
$$

so $D(E)=\infty$.

REMARK. Godun [5] has achieved the proof that $D(E) \geq 2$ for every non-reflexive Banach space, by showing the following stronger result. For every non-reflexive Banach space $E$ and each $\varepsilon>0$ there exists an equivalent norm $\|\cdot\|$ on $E$ such that there exists no projection $p$ of norm $\|p\|<2-\varepsilon$ of $E^{* *}$ onto $K(E)$, the canonical image of $E$ in $E^{* *}$. Since Godun [5] did not mention the paper [2], let us observe that the weaker result in which $\|p\|<2-\varepsilon$ is replaced by $\|p\|=1$, had been proved in [2], Theorem 2.I (giving an affirmative answer to a problem of Davis and Johnson [1]) and that the above result of Godun solves Problem 2.1 of [2]. Also, in [2] it was observed that for any equivalent norm \|\|$\|$ on a quasi-reflexive space $E$ of order $I$ (that is, with $\operatorname{dim} E^{* *} / K(E)=2$ ) and for any $\varepsilon>0$ there exists a projection $p$ of $E^{* *}$ onto $k(E)$ of norm $\|p\|<2+\varepsilon$. However, the following problem remains open:

PROBLEM. Let $E$ be a non-reflexive Banach space. Does there exist an equivalent norm \|\|$\|$ on $E$ such that there exists no projection $p$ of norm $\|p \mid\| \leq 2$ of $E^{* *}$ onto $K(E)$ ?

\section{References}

[1] William J. Davis and William B. Johnson, "A renorming of nonreflexive Banach spaces", Proc. Amer. Math. Soc. 37 (1973), 486-488.

[2] Dick van Dulst and Ivan Singer, "On Kadec-Klee norms on Banach spaces", Studia Math. 54 (1975-1976), 205-211.

[3] Per Enflo, "A counterexample to the approximation problem in Banach spaces", Acta Math. 130 (1973), 309-317. 
[4] T. Figiel and W.B. Johnson, "The approximation property does not imply the bounded approximation property", Proc. Amer. Math. Soc. 41 (1973), 197-200.

[5] 5.В. Годун [B.V. Godun], "Энвивалентные нормы на нерефленснвных пространствах" [Equivalent norms on nonreflexive spaces], Dokl. Akad. Nauk SSSR 236 (1977), 18-20.

[6] Alexandre Grothendieck, Produits tensoriels topologiques et espaces nucléaires (Memoirs Amer. Math. Soc. 16. American Mathematical Society, Providence, Rhode Island, 1955).

[7] W.B. Johnson, H.P. Rosenthal and M. Zippin, "On basis, finite dimensional decompositions and weaker structures in Banach spaces", Israel J. Math. 9 (1971), 488-506.

[8] Joram Lindenstrauss, "On James's paper 'Separable conjugate spaces'", Israel J. Math. 9 (1971), 279-284.

Institutul Nafional pentru Creafie Ştiinfifică şi Tehnică, Bucureşti,

Romania;

Institutul de Matematica, Bucureşti, Romania. 